\title{
The CT Swirl Sign Is Associated with Hematoma Expansion in Intracerebral Hemorrhage
}

\author{
(DD. Ng, (D) Churilov, (DP. Mitchell, DR. Dowling, and (D)B. Yan
}

\begin{abstract}
BACKGROUND AND PURPOSE: Hematoma expansion is an independent determinant of poor clinical outcome in intracerebral hemorrhage. Although the "spot sign" predicts hematoma expansion, the identification requires CT angiography, which limits its general accessibility in some hospital settings. Noncontrast CT, without the need for CT angiography, may identify sites of active extravasation, termed the "swirl sign." We aimed to determine the association of the swirl sign with hematoma expansion.
\end{abstract}

MATERIALS AND METHODS: Patients with spontaneous intracerebral hemorrhage between 2007 and 2014 who underwent an initial and subsequent noncontrast CT at a single center were retrospectively identified. The swirl sign, on noncontrast CT, was defined as iso- or hypodensity within a hyperdense region that extended across 2 contiguous 5-mm axial CT sections.

RESULTS: A total of 212 patients met the inclusion criteria. The swirl sign was identified in 91 patients with excellent interobserver agreement $(\kappa=0.87)$. The swirl sign was associated with larger initial hematoma $(P<.001)$ and earlier initial $C T(P<.001)$ and hematoma expansion $(P=.028)$. Multivariable regression modeling demonstrated that if one assumed similar initial hematoma volume, onset-to-first scan, and time between CT scans, the median absolute hematoma growth was $5.77 \mathrm{~mL}(95 \% \mathrm{Cl}, 2.37-9.18 \mathrm{~mL} ; P=.001)$ and relative growth was $35.6 \%(95 \% \mathrm{Cl}, 18.5 \%-52.6 \% ; P<.001)$ higher in patients with the swirl sign compared with those without.

CONCLUSIONS: The NCCT swirl sign was reliably identified and is associated with hematoma expansion. We propose that the swirl sign be included in risk stratification of intracerebral hemorrhage and considered for inclusion in clinical trials.

ABBREVIATIONS: $I \mathrm{CH}=$ intracerebral hemorrhage; IQR = interquartile range

$\mathbf{T}$ he annual incidence of intracerebral hemorrhage (ICH) worldwide is estimated at 4 million with a large proportion occurring in low- to middle-income countries. ${ }^{1} \mathrm{ICH}$ confers a poorer prognosis compared with ischemic stroke with morbidity and mortality approaching $50 \%$ at 30 days. $^{2}$

Established prognostic factors include initial hematoma volume, age, low Glasgow Coma Scale score, and shorter time from onset to presentation. ${ }^{3,4}$ These factors may not reflect the dynamic nature of hematoma evolution and expansion, which are important in the clinical outcome. ${ }^{5}$ Hematoma growth due to active extravasation and rebleeding has been reported in $38 \%$ of patients after initial CT. ${ }^{6}$ Hematoma expansion has been shown

Received July 31, 2017; accepted September 19.

From the Department of Radiology (D.N., P.M., R.D., B.Y.), and Melbourne Brain Centre (B.Y.), University of Melbourne, Royal Melbourne Hospital, Parkville, Victoria, Australia; and Statistics and Decision Analysis (L.C.), Florey Institute of Neuroscience and Mental Health, Heidelberg, Victoria, Australia.

Please address correspondence to Bernard Yan, MD, Melbourne Brain Centre, Royal Melbourne Hospital, 300 Grattan St, Parkville Victoria 3050 Australia; e-mail: bernard.yan@mh.org.au

http://dx.doi.org/10.3174/ajnr.A5465 to be an independent determinant of poorer clinical outcome. ${ }^{5}$ Consequently, identification of patients at increased risk of hematoma expansion and the development of strategies to limit hematoma growth have been a focus of clinical trials. ${ }^{1,5,7-10}$

Current Phase II trials, the Spot Sign and Tranexamic Acid On Preventing ICH Growth-AUStralasia Trial (STOP-AUST), "Spot Sign" Selection of Intracerebral Hemorrhage to Guide Hemostatic Therapy (SPOTLIGHT), Intensive Blood Pressure Reduction in Acute Cerebral Hemorrhage Trial 2 (INTERACT 2), and Antihypertensive Treatment of Acute Cerebral Hemorrhage (ATACH II), investigated the reduction of hematoma growth using tranexamic acid, recombinant factor VIIa, and blood pressure control. ${ }^{1,9-11}$ STOP-AUST and SPOTLIGHT used the CTA "spot sign" as a basis for patient selection. The spot sign is a biomarker for active hemorrhage and independently predicts relative and absolute hematoma expansion. ${ }^{12-17}$ Currently, CT angiography is widely performed in cases in which an ischemic stroke is suspected, while it is less commonly performed in the hemorrhagic subtype. Limitations for contrast administration include nephrotoxicity, contraindications in moderate-to-severe renal impair- 

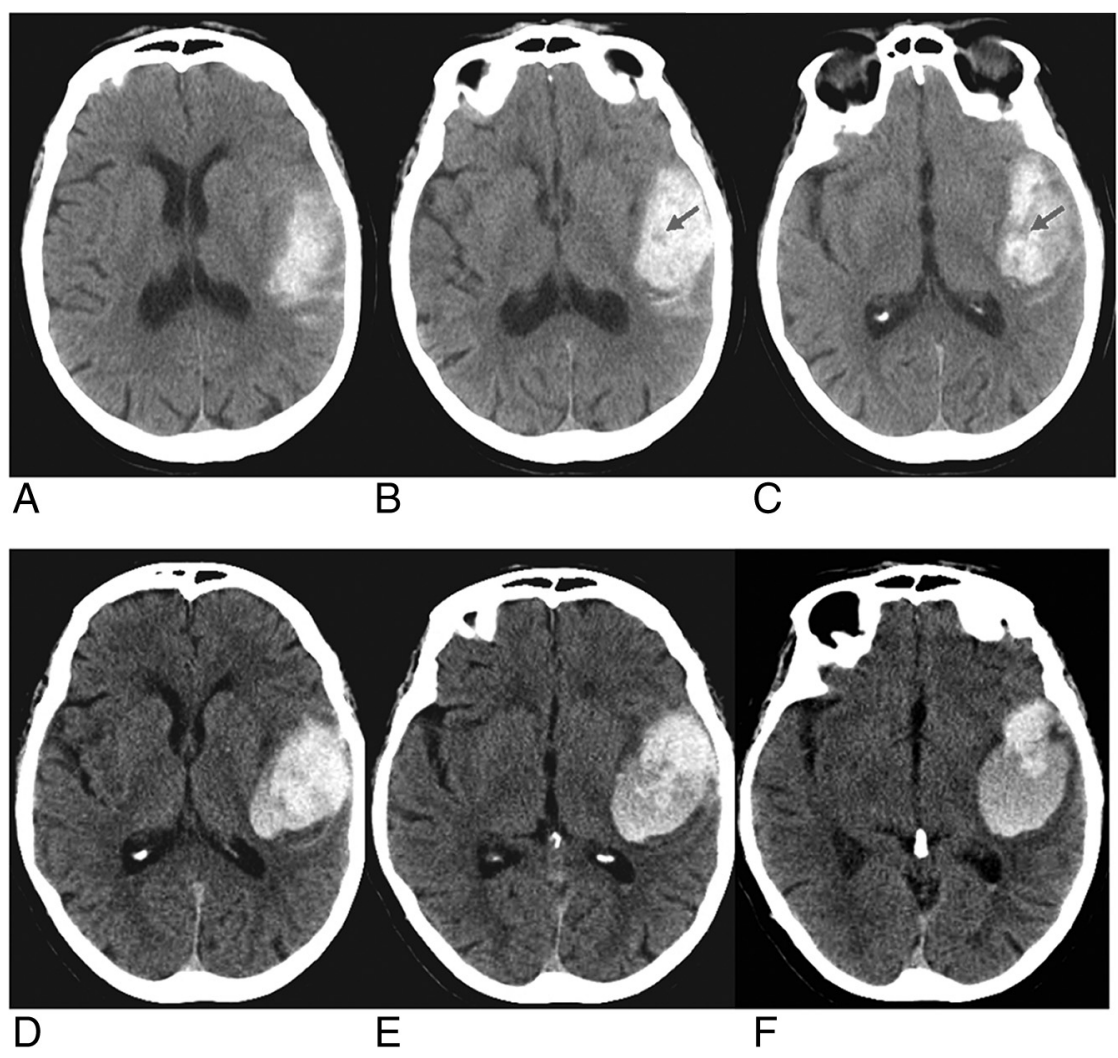

FIGURE. Noncontrast brain CT of a 73-year-old woman who presented with right-sided weakness. Initial brain $C T(A-C)$ demonstrates a left parietal hematoma measuring $33 \mathrm{~mL}$, demonstrating hypodense hematoma with hypodense foci, the swirl sign. Follow-up CT $(D-F)$ performed 8 hours later demonstrates increased hematoma volume, $46 \mathrm{~mL}$.

ment, prior contrast reaction, and access and availability of staff or infrastructure in rural areas, which preclude the use of this prognostic tool. ${ }^{18,19}$ Thus, there remains a role for noncontrast $\mathrm{CT}$ in identifying patients at risk for hematoma growth.

The "swirl sign" has been previously described as a hypo- or isodensity within a region of a hyperdensity that correlates with active hemorrhage on surgical evacuation. ${ }^{20}$ It is reproducible and an independent predictor of 1-month mortality and functional outcomes. ${ }^{21}$ The utility of the swirl sign in predicting hematoma growth has not been thoroughly investigated. ${ }^{21,22} \mathrm{We}$ aimed to further define the swirl sign and evaluate its value in predicting hematoma expansion. With the CT swirl sign, it may be possible to identify patients with intracerebral hemorrhage most likely to have hematoma expansion in the absence of CT angiography and for whom hemostatic therapy will be of benefit.

\section{MATERIALS AND METHODS \\ Study Population}

This was a retrospective analysis of all patients who presented to the Royal Melbourne Hospital, a quaternary stroke referral center, with a spontaneous intracerebral hemorrhage diagnosed between September 2007 and August 2014. All potential cases were identified with our prospective stroke data base. The diagnosis was made on CT by a neuroradiologist. The exclusion criteria were an absence of an initial CT at our institution or a repeat CT scan, imaging performed 96 hours after symptom onset (including interhospital transfers) or subsequent CT performed 96 hours after initial CT, surgical decompression between CT1 and CT2 (for which the hematoma volume on subsequent CT could not be assessed), concurrent vascular abnormalities (intracranial aneurysms, arteriovenous malformations, cavernomas, hemangiomas), trauma, or tumor. The study adhered to the principles of the Declaration of Helsinki and was approved by the Melbourne Health Human Research Ethics Committee.

\section{Demographic and Clinical Data}

Demographic and clinical data were obtained via a prospectively organized stroke data base compiled after review of patient medical records. Parameters included age, sex, history (previous stroke/ transient ischemic attack, diabetes mellitus, hypertension), medications, time of onset, and initial examination findings (Glasgow Coma Scale, initial systolic blood pressure). The modified Rankin Scale score on admission was also calculated. Interventions, including warfarin reversal, were also noted.

Biochemical results including hemoglobin level, platelet level, and coagulation profile (international normalized ratio, activated partial thromboplastin time, fibrinogen levels) were obtained using the electronic pathology data base of Melbourne Health, clinical information service.

\section{Radiologic Findings}

Initial noncontrast CT scans were reviewed independently by the primary investigator (D.N., 6 months of neuroimaging experience) and an interventional neuroradiologist (R.D., 18 years of neuroimaging experience) for the presence or absence of the swirl sign. They were blinded to clinical data and the presence of hematoma enlargement. The swirl sign was defined as an area of low density (30-50 HU, hypo- or isodense to brain parenchyma) surrounded by a hyperdense region (Figure). The hypodensity had to extend across 2 contiguous $5-\mathrm{mm}$ axial CT sections. The sign represents active hemorrhage within an area of acute hematoma.

Hematoma volume, location (supra- or infratentorial in relation to the tentorium cerebelli), presence of midline shift, and intraventricular extension were obtained from previously published data in our stroke data base. ${ }^{23}$ Hematoma growth was calculated as absolute (hematoma volume measured on CT2-CT1) and relative values (hematoma volume measured on CT2-CT1) / Volume on CT1).

\section{Statistical Analysis}

Statistical analysis was conducted by using STATA 13 IC (StataCorp, College Station, Texas). $P$ values $<.05$ were indicative of statistical significance.

Patient characteristics of those included and excluded from 
Table 1: Comparison of included and excluded patients

\begin{tabular}{|c|c|c|c|}
\hline Patient Characteristics & $\begin{array}{l}\text { Patients with } \\
\text { Spontaneous } \\
\mathrm{ICH}(n=212)\end{array}$ & $\begin{array}{l}\text { Patients Excluded } \\
\qquad(n=460)\end{array}$ & $P$ Value \\
\hline Age (median) (IQR) (yr) & $68(61-77.5)$ & $75(64.5-83)$ & $<.001$ \\
\hline Male sex (No.) (\%) & $86(40.6)$ & $210(45.7)$ & .21 \\
\hline Time to initial CT (median) (IQR) (hr) & $4.5(2.29-17.5)$ & $4.4(2.0-11.6)[n=299]$ & .06 \\
\hline Time to second CT (median) (IQR) (hr) & $11.92(5.38-29.27)$ & $19.9(4.6-56.64)[n=287]$ & .005 \\
\hline \multicolumn{4}{|l|}{ PMHx (No.) (\%) } \\
\hline Hypertension & $166(78.3)$ & $329(71.8)$ & .09 \\
\hline Hypercholesterolemia & $68(32.1)$ & $114(30.6)$ & .71 \\
\hline Statin use & $68(32.1)$ & $145(31.5)$ & .92 \\
\hline Diabetes & $54(25.6)$ & $108(23.8)$ & .32 \\
\hline Ischemic heart disease & $39(18.4)$ & $79(17.6)$ & .83 \\
\hline Atrial fibrillation & $47(22.2)$ & $93(20.7)$ & .68 \\
\hline \multicolumn{4}{|l|}{ Previous stroke } \\
\hline Transient ischemic attack & $13(6.2)$ & $29(6.5)$ & 1.00 \\
\hline Ischemic stroke $(n=207)$ & $21(9.9)$ & $43(11.5)$ & .68 \\
\hline Hemorrhagic stroke & $12(5.8)$ & $35(9.4)$ & .15 \\
\hline \multicolumn{4}{|l|}{ Anticoagulant (No.) (\%) } \\
\hline Warfarin & $42(19.8)$ & $87(18.9)$ & .83 \\
\hline Antiplatelet & $70(33.0)$ & $164(35.6)$ & .54 \\
\hline GCS on presentation (median) (IQR) & $14(11-15)[n=207]$ & $14(10-15)[n=355]$ & .52 \\
\hline \multicolumn{4}{|l|}{ Radiologic (No.) (\%) } \\
\hline Location supratentorial & $173(81.6)$ & $309(67.2)$ & .42 \\
\hline IV extension $(n=326)$ & $88(41.5)$ & $60(52.6)$ & .06 \\
\hline Midline shift $(n=499)$ & $78(36.8)$ & $142(49.5)$ & .006 \\
\hline
\end{tabular}

Note:-GCS indicates Glasgow Coma Scale; PMHx, past medical history; IV, intraventricular.

Table 2: Comparison of patients positive and negative for the swirl sign

\begin{tabular}{|c|c|c|c|}
\hline Patient Characteristics & $\begin{array}{l}\text { Swirl Sign -ve } \\
\quad(n=121)\end{array}$ & $\begin{array}{c}\text { Swirl +ve } \\
(n=91)\end{array}$ & $P$ Value \\
\hline Age (median) (IQR) (yr) & $69(51-76)$ & $68(51-72)$ & .4 \\
\hline Male sex (No.) (\%) & $50(41.3)$ & $36(39.6)$ & .89 \\
\hline Time to initial CT (median) (IQR) (hr) & $16.1(1.1-95.3)$ & $8.0(0.37-94)$ & $<.001$ \\
\hline Time to second CT (median) (IQR) (hr) & $21.0(10.3-28.5)$ & $16.5(5.9-29.5)$ & .12 \\
\hline Initial hematoma volume (median) (IQR) (mL) & $8.56(3.83-14.95)$ & $23.2(11.65-43.3)$ & $<.001$ \\
\hline \multicolumn{4}{|l|}{ PMHx (No.) (\%) } \\
\hline Hypertension & $99(81.8)$ & $67(73.6)$ & .18 \\
\hline Hypercholesterolemia & $45(37.3)$ & $23(25.3)$ & .08 \\
\hline Statin use & $40(33.1)$ & $28(30.8)$ & .77 \\
\hline Diabetes & $33(27.5)$ & $21(23.1)$ & .52 \\
\hline Ischemic heart disease & $21(17.4)$ & $18(19.8)$ & .72 \\
\hline Atrial fibrillation & $21(17.4)$ & $26(28.6)$ & .06 \\
\hline \multicolumn{4}{|l|}{ Previous stroke } \\
\hline Transient ischemic attack & $9(7.5)$ & $4(4.4)$ & .40 \\
\hline Ischemic stroke $(n=207)$ & $10(8.5)$ & $11(12.2)$ & .49 \\
\hline Hemorrhagic stroke & $8(6.8)$ & $4(4.4)$ & .56 \\
\hline \multicolumn{4}{|l|}{ Anticoagulant (No.) (\%) } \\
\hline Warfarin & 19 (15.7) & $23(25.3)$ & .12 \\
\hline Antiplatelet & $42(34.7)$ & $28(30.8)$ & .56 \\
\hline GCS on presentation (median) (IQR) $(n=207)$ & $14(12-15)$ & 13 (11-14) & .018 \\
\hline INR (median) (IQR) & $1.1(1.0-1.2)$ & $1.1(1.0-1.4)$ & .13 \\
\hline \multicolumn{4}{|l|}{ Radiologic (No.) (\%) } \\
\hline Location supratentorial & $95(78.5)$ & $78(85.7)$ & .21 \\
\hline IV extension $(n=145)$ & $50(63.3)$ & $38(57.6)$ & 1.00 \\
\hline Midline shift & $27(22.3)$ & $51(56.0)$ & $<.001$ \\
\hline
\end{tabular}

Note:-INR indicates international normalized ratio; -ve, negative; +ve, positive; PMHx, past medical history; GCS, Glasgow Coma Scale; IV, intraventricular.

the study, those positive or negative for the swirl sign, and those with the swirl sign with and without hematoma growth were summarized as median and interquartile range (IQR) for continuous variables and counts and proportions for categoric variables and were compared using the Wilcoxon-Mann-Whitney and Fisher exact tests, respectively.

Interobserver agreement for the presence of the swirl sign was estimated using the Cohen $\kappa$ score, with a $\kappa$ of $0.81-1.00$ corresponding to almost perfect agreement; 0.61-0.80, substantial; 0.41-0.60, moderate; 0.21-0.40, fair; $0.00-0.20$, slight; and $<0.00$, poor agreement. ${ }^{24}$

To investigate the association between the presence or absence of the swirl sign and hematoma growth, we created 2 kinds of models in which hematoma growth was expressed as either an absolute or a relative change. Multiple median regression was performed with hematoma growth as an output; the swirl sign as a factor; and hematoma volume on the initial $\mathrm{CT}$, time difference between the first and second $\mathrm{CT}$, and onset of symptoms to the first CT, as covariates. The resulting models were further validated by running linear leastsquares regression models with the same input but with the output transformed by using fifth-root transformation to ensure satisfaction of model assumptions.

\section{RESULTS}

\section{Patient Characteristics}

A total of 672 patients were treated for spontaneous ICH and had an assessable initial CT scan between October 2007 and August 2014. Four hundred sixty were excluded from the analysis, including 405 who did not have a repeat CT, 30 in whom the interval between onset and initial CT exceeded 4 days, 8 in whom the interval between CT1 and CT2 exceeded 4 days, 15 who underwent surgical decompression between CT1 and CT2 (13 demonstrated the swirl sign on initial CT), and 2 who could not be assessed due to poor image quality.

A total of 212 patients met the inclusion criteria, including 86 (40.6\%) men with a median age of 68 years (IQR, 6177.5 years).

Previous stroke was documented in $33(15.9 \%)$ patients with a hemorrhagic etiology in $12(5.8 \%)$ and ischemic in 21 $(9.9 \%)$. Clinical risk factors for $\mathrm{ICH}$ included hypertension ( $n=166,78.3 \%$ ), antiplatelet use 70 (33\%), and anticoagulant use 42 (19.8\%). On presentation, patients had a median international normalized ratio of 1.1 (IQR, 1.0-1.2) and a Glasgow Coma Scale score of 14 (IQR, 12-15).

The median time from symptom onset to initial CT was 4.5 hours (IQR, 2.29-17.5 hours). The hemorrhage was supratentorial in 173 $(81.6 \%)$ patients, associated with intraventricular extension in $88 /$ $145(60.7 \%)$, and midline shift in $78(36.8 \%)$.

\section{Comparison between Included and Excluded Patients}

The excluded patients were older $(P<.001)$ and were more likely to have midline shift $(P=.006)$ (Table 1$)$. No statistically signif- 
Table 3: Comparison of patients positive for the swirl sign with and without hematoma growth

\begin{tabular}{lccc}
\multicolumn{1}{c}{ Patient Characteristics } & $\begin{array}{c}\text { Increased } \\
\text { Hematoma } \\
\text { Volume }(\boldsymbol{n}=65)\end{array}$ & $\begin{array}{c}\text { Stable/Reduced } \\
\text { Hematoma } \\
\text { Volume }(\boldsymbol{n}=26)\end{array}$ & $\boldsymbol{P}$ Value \\
\hline Age (median) (IQR) (yr) & $71(63-76)$ & $66(55-72)$ & .06 \\
Male sex (No.) (\%) & $25(38.5)$ & $11(42.3)$ & .81 \\
Time to initial CT (median) (IQR) (hr) & $3(1.7-6.6)$ & $4(2.6-12.7)$ & .01 \\
Time to second CT (median) (IQR) (hr) & $12(4.2-23.8)$ & $26.8(14.7-36.0)$ & $<.001$ \\
Initial hematoma volume (median) (IQR) (mL) & $24(12.2-49.6)$ & $21.6(10.3-38.5)$ & .37 \\
PMHx (No.) (\%) & & & \\
Hypertension & $46(70.7)$ & $21(80.8)$ & .43 \\
Hypercholesterolemia & $17(26.2)$ & $6(23.8)$ & 1.00 \\
Statin use & $22(33.9)$ & $6(23.8)$ & .45 \\
Diabetes & $16(24.6)$ & $5(19.2)$ & .78 \\
Ischemic heart disease & $15(23.1)$ & $3(11.5)$ & .26 \\
Atrial fibrillation & $23(35.4)$ & $3(11.5)$ & .018 \\
Previous stroke & & & \\
Transient ischemic attack & $3(4.6)$ & $1(3.9)$ & 1.00 \\
Ischemic stroke ( $=207)$ & $8(12.3)$ & $3(11.5)$ & 1.00 \\
Hemorrhagic stroke & $3(4.6)$ & $1(3.9)$ & 1.00 \\
Anticoagulant (No.) (\%) & & & \\
Warfarin & $22(33.9)$ & $1(3.9)$ & .003 \\
Antiplatelet & $22(33.9)$ & $6(23.8)$ & .45 \\
GCS on presentation (median) (IQR) & $14(9.8-14)$ & $13(11-14)$ & .13 \\
INR (median) (IQR) & $1.2(1.0-1.8)$ & $1.1(1.0-1.1)$ & .01 \\
Radiologic (No.) (\%) & & & .75 \\
Location supratentorial & $55(84.6)$ & $23(88.5)$ & .35 \\
IV extension & $17(26.2)$ & $11(42.3)$ & .16 \\
Midline shift & $33(50.8)$ & $18(69.2)$ & \\
\hline Note & & & \\
\hline
\end{tabular}

Note:-GCS indicates Glasgow Coma Scale; PMHx, past medical history; IV, intraventricular; INR, international normalized ratio.

Table 4: Multiple median regression model (follow-up volume)

\begin{tabular}{lccc}
\hline \multicolumn{1}{c}{ Follow-Up Volume } & $\begin{array}{c}\text { Median Absolute } \\
\text { Growth }(\mathbf{m L})\end{array}$ & $\mathbf{P}$ Value & $\mathbf{9 5 \% ~ C l}$ \\
\hline Swirl sign & 5.77 & .001 & 2.37 to 9.18 \\
Initial hematoma volume $(\mathrm{mL})$ & 1.01 & .000 & 0.95 to 1.06 \\
Time between CT1 and CT2 (hr) & -0.07 & .028 & -0.12 to -0.007 \\
Time between onset and CT1 (hr) & -0.0003 & .251 & -0.0008 to 0.0002 \\
\hline
\end{tabular}

Table 5: Multiple median regression model (relative hematoma volume change)

\begin{tabular}{|c|c|c|c|}
\hline Relative Hematoma Volume Change & $\begin{array}{c}\text { Median Relative } \\
\text { Growth (\%) }\end{array}$ & $P$ Value & $95 \% \mathrm{Cl}$ \\
\hline Swirl sign & 35.6 & $<.001$ & 18.5 to 52.6 \\
\hline Initial hematoma volume (mL) & -0.23 & .158 & -0.56 to 0.09 \\
\hline Time between CT1 and CT2 (hr) & -0.42 & .008 & -0.73 to -0.11 \\
\hline Time between onset and CTI (hr) & 0.0 & .424 & -0.01 to 0.00 \\
\hline
\end{tabular}

$61 \%-80.4 \%$ ) patients with positive swirl signs compared with 33 (27\%; $95 \%$ CI, $19.6 \%-36.1 \%$ ) of those without the swirl sign (Table 2). On univariate analysis, no significant difference in the age, sex, presence of hypertension, diabetes, previous stroke of any cause, ischemic heart disease, or antiplatelet use was observed between those with and those without the swirl sign (Table 2). No statistically significant association between the presence of the swirl sign and the initial Glasgow Coma Scale score, initial mRS, or international normalized ratio was observed (Table 2). No statistically significant association between the presence of the swirl sign and time of symptom onset to initial CT $(P=.08)$, atrial fibrillation $(P=.06)$, or anticoagulant use $(P=.12)$ was observed (Table 2$)$.

\section{Association between the Swirl Sign and Hematoma Growth}

Patients positive for the swirl sign had larger initial hematoma volumes $(P<$ $.001)$ and a shorter time between symptom onset and initial CT $(P<.001)$ and were more likely to have midline shift $(P<.001)$ compared with those without (Table 2). No statistically significant differences in the hemorrhage location or time taken for repeat imaging were observed.

Patients with the swirl sign and hematoma growth were more likely to have atrial fibrillation $(P=.018)$, be on warfarin $(P=.003)$ with a higher international normalized ratio $(P=.01)$, and have a shorter interval between onset and CT1 $(P=.01)$ and between CT1 and CT2 $(P<.001)$ (Table 3$)$.

Multivariable regression modeling demonstrated that if we assumed similar initial hematoma volume, onset-to-first

icant differences in sex, time to initial CT, traditional cardiovascular risk factors, previous stroke, and use of anticoagulants between included and excluded patients were observed (Table 1).

\section{Interobserver Agreement}

A random sample of $60 \mathrm{CT}$ scans demonstrated excellent interobserver agreement in identifying the swirl sign $(\kappa=0.87 ; 95 \% \mathrm{CI}$, 0.75-0.99). There was disagreement about the presence of the swirl sign in 4 of 60 patients. Three of these patients had a small hematoma volume $(<10 \mathrm{~mL})$.

\section{The Swirl Sign and Clinical Variables}

The swirl sign was present in 91 (42.9\%; 95\% CI, 36.2\%-49.9\%) patients. Hematoma expansion occurred in 65 (71\%; 95\% CI, scan time, and time between CT scans, the median absolute hematoma growth was $5.77 \mathrm{~mL}(95 \% \mathrm{CI}, 2.37-9.18 \mathrm{~mL} ; P=.001)$ higher and the median relative hematoma growth was $35.6 \%$ (95\% CI, 18.5\%-52.6\%; $P<.001$ ) higher in patients with the swirl sign compared with those without (Tables 4 and 5).

Qualitatively similar associations were observed on a robustness analysis using least-squares linear regression models with the same input and the output transformed by using fifth-root transformation.

\section{DISCUSSION}

Our retrospective study demonstrates that if we assume similar initial hematoma volume and time between CT scans, the median 
absolute hematoma growth $(5.77 \mathrm{~mL} ; 95 \% \mathrm{CI}, 2.37-9.18 \mathrm{~mL} ; P=$ $.001)$ and relative hematoma growth (35.6\%; 95\% CI, $18.5 \%-$ $52.6 \%$; $P<.001)$ in patients with the swirl sign were higher than in those without. The swirl sign was common and present in $43 \%$ $(91 / 212)$ of our study population.

Hematoma evolution in $\mathrm{ICH}$ is a dynamic process that begins as a primary hemorrhagic insult. Multiple studies have shown that a significant proportion of patients (38\%) undergo hematoma expansion on repeat CT imaging. ${ }^{6}$ The currently recognized clinical prognostic factors (including initial hematoma volume, neurologic deficit at presentation, age, and infratentorial location) do not directly reflect the dynamic nature of hematoma evolution. Hematoma growth is thought to be due to active hemorrhage and rebleeding and has been shown to be an independent determinant of mortality and morbidity. ${ }^{5}$ Early identification and hematoma limitation have become a treatment goal. ${ }^{5}$

The swirl sign on noncontrast CT is iso- or hypodense within a hyperdense region and is thought to represent an area of active hemorrhage within an acute hematoma. ${ }^{20}$ The swirl sign was first described in traumatic extra-axial hematomas. ${ }^{25}$ Greenberg et $\mathrm{al}^{26}$ and Al-Nakshabandi ${ }^{20}$ documented that following surgical exploration, the swirl sign correlated with areas of active hemorrhage in subdural hematomas.

Selariu et $\mathrm{al}^{21}$ demonstrated that the swirl sign was reproducible and correlated with increased mortality and poorer functional outcomes. The swirl sign was present in 30\% (61/203) of the study population. The patients positive for the swirl sign were more likely to demonstrate midline shift and intraventricular hematoma extension and have larger initial hematoma volumes (mean, 52 versus $15 \mathrm{~mL}$ ). Our study supports these findings.

Gökçe et $\mathrm{al}^{22}$ studied the CT findings of 45 patients with ICH on antihypertensive and anticoagulant agents. The median initial hematoma volume was $46 \mathrm{~mL}$, with the swirl sign identified in 32 (71\%) patients. All patients with the swirl sign demonstrated hematoma enlargement on repeat imaging. ${ }^{22}$ Kim et $\mathrm{al}^{27}$ found that the swirl sign, among other prognostic factors, was associated with increased mortality but did not independently predict mortality and hematoma growth.

To our knowledge, no previous study has specifically examined the association between the swirl sign and hematoma growth. The CTA spot sign has been extensively investigated and prospectively validated as an independent determinant of hematoma expansion, morbidity, and mortality. ${ }^{3,12,13,28}$ Currently, the spot sign is the imaging benchmark for hematoma growth in large national trials investigating hemostatic agents. ${ }^{1}$ However, CT angiography is contraindicated in patients with prior contrast reactions and renal impairment and has reduced availability. Identification of baseline CT predictors of ICH expansion is important to aid selection in hemostatic trials when CTA is not possible. This study supports the swirl sign as a reproducible marker of hematoma growth that can be used as an adjunct to the spot sign.

The strengths of our study include the study population size and length of the recruitment period. The swirl sign was further defined to improve reproducibility and attempt to reduce the impact of imaging artifacts.

Limitations of the study include a selection bias, which may have been introduced by our exclusion criteria. The excluded pa- tients were older $(P<.001)$ and more likely to demonstrate midline shift $(P=.006)$ on initial CT. These features probably reflect the effects of a larger hematoma, and it is postulated that a higher proportion of these patients would be swirl-positive. Thus, this understates the incidence and strength of the association of the swirl sign and hematoma growth. In addition, a number of patients were transferred from another hospital, resulting in a longer time to imaging, which may underestimate the degree of active hemorrhage. Furthermore, the impetus for repeat CT imaging was dependent on the degree of clinical concern of the treating neurology team. Consequently, the interval between initial and subsequent CT varied; this difference may underestimate the degree of hematoma expansion in delayed imaging due to clot retraction.

\section{CONCLUSIONS}

The noncontrast CT swirl sign was reliably identified with excellent interrater agreement. The presence of the swirl sign was associated with hematoma expansion. We propose that the swirl sign should be included in the risk stratification of intracerebral hemorrhage and should be considered for inclusion in clinical trials.

\section{REFERENCES}

1. Meretoja A, Churilov L, Campbell BC, et al. The spot sign and tranexamic acid on preventing ICH growth: AUStralasia Trial (STOP-AUST) — protocol of a phase II randomized, placebo-controlled, double-blind, multicenter trial. Int J Stroke 2014;9:519-24 CrossRef Medline

2. Flaherty ML, Haverbusch M, Sekar P, et al. Long-term mortality after intracerebral hemorrhage. Neurology 2006;66:1182-86 CrossRef Medline

3. Hemphill JC 3rd, Bonovich DC, Besmertis L, et al. The ICH score: a simple, reliable grading scale for intracerebral hemorrhage. Stroke 2001;32:891-97 CrossRef Medline

4. Curtze S, Strbian D, Meretoja A, et al. Higher baseline international normalized ratio value correlates with higher mortality in intracerebral hemorrhage during warfarin use. Eur J Neurol 2014;21: 616-22 CrossRef Medline

5. Davis SM, Broderick J, Hennerici M, et al; Recombinant Activated Factor VII Intracerebral Hemorrhage Trial Investigators. Hematoma growth is a determinant of mortality and poor outcome after intracerebral hemorrhage. Neurology 2006;66:1175-81 Medline

6. Brott T, Broderick J, Kothari R, et al. Early hemorrhage growth in patients with intracerebral hemorrhage. Stroke 1997;28:1-5 CrossRef Medline

7. Edavettal M, Rogers A, Rogers F, et al. Prothrombin complex concentrate accelerates international normalized ratio reversal and diminishes the extension of intracranial hemorrhage in geriatric trauma patients. Am Surg 2014;80:372-76 Medline

8. Romero JM, Heit JJ, Delgado Almandoz JE, et al. Spot sign score predicts rapid bleeding in spontaneous intracerebral hemorrhage. Emerg Radiol 2012;19:195-202 CrossRef Medline

9. Hill MD, Muir KW. INTERACT-2: should blood pressure be aggressively lowered acutely after intracerebral hemorrhage? Stroke 2013; 44:2951-52 CrossRef Medline

10. Gladstone D. "Spot Sign" Selection of Intracerebral Hemorrhage to Guide Hemostatic Therapy (SPOTLIGHT). 2011. https://clinicaltrials. gov/ct2/show/NCT01359202. Accessed May 1, 2017

11. Qureshi AI, Palesch YY. Antihypertensive Treatment of Acute Cerebral Hemorrhage (ATACH) II: design, methods, and rationale. Neurocrit Care 2011;15:559-76 CrossRef Medline

12. Huynh TJ, Demchuk AM, Dowlatshahi D, et al; PREDICT/Sunny- 
brook ICH CTA Study Group. Spot sign number is the most important spot sign characteristic for predicting hematoma expansion using first-pass computed tomography angiography: analysis from the PREDICT study. Stroke 2013;44:972-77 CrossRef Medline

13. Demchuk AM, Dowlatshahi D, Rodriguez-Luna D, et al; PREDICT/ Sunnybrook ICH CTA study group. Prediction of haematoma growth and outcome in patients with intracerebral haemorrhage using the CT-angiography spot sign (PREDICT): a prospective observational study. Lancet Neurol 2012;11:307-14 CrossRef Medline

14. Delgado Almandoz JE, Yoo AJ, Stone MJ, et al. Systematic characterization of the computed tomography angiography spot sign in primary intracerebral hemorrhage identifies patients at highest risk for hematoma expansion: the spot sign score. Stroke 2009;40:29943000 CrossRef Medline

15. Rost NS, Smith EE, Chang Y, et al. Prediction of functional outcome in patients with primary intracerebral hemorrhage: the FUNC score. Stroke 2008;39:2304-09 CrossRef Medline

16. Broderick JP, Diringer MN, Hill MD, et al; Recombinant Activated Factor VII Intracerebral Hemorrhage Trial Investigators. Determinants of intracerebral hemorrhage growth: an exploratory analysis. Stroke 2007;38:1072-75 CrossRef Medline

17. Godoy DA, Piñero G, Di Napoli M. Predicting mortality in spontaneous intracerebral hemorrhage: can modification to original score improve the prediction? Stroke 2006;37:1038-44 CrossRef Medline

18. Leys D, Cordonnier C, Debette S, et al; Executive committee of the European Stroke Initiative (EUSI). Facilities available in French hospitals treating acute stroke patients: comparison with 24 other European countries. J Neurology 2009;256:867-73 CrossRef Medline
19. Goldstein LB. Statewide hospital-based stroke services in North Carolina: changes over 10 years. Stroke 2010;41:778-83 CrossRef Medline

20. Al-Nakshabandi NA. The swirl sign. Radiology 2001;218:433 CrossRef Medline

21. Selariu E, Zia E, Brizzi M, et al. Swirl sign in intracerebral haemorrhage: definition, prevalence, reliability and prognostic value. BMC Neurol 2012;12:109 CrossRef Medline

22. Gökçe E, Beyhan M, Acu B. Evaluation of oral anticoagulant-associated intracranial parenchymal hematomas using CT findings. Clin Neuroradiol 2015;25:151-59 CrossRef Medline

23. Ma M, Meretoja A, Churilov L, et al. Warfarin-associated intracerebral hemorrhage: volume, anticoagulation intensity and location. J Neurol Sci 2013;332:75-79 CrossRef Medline

24. Landis JR, Koch GG. The measurement of observer agreement for categorical data. Biometrics 1977;33:159-74 CrossRef Medline

25. Zimmerman RA, Bilaniuk LT. Computed tomographic staging of traumatic epidural bleeding. Radiology 1982;144:809-12 CrossRef Medline

26. Greenberg J, Cohen WA, Cooper PR. The "hyperacute" extra-axial intracranial hematoma: computed tomographic findings and clinical significance. Neurosurgery 1985;17:48-56 CrossRef Medline

27. Kim J, Smith A, Hemphill JC 3rd, et al. Contrast extravasation on CT predicts mortality in primary intracerebral hemorrhage. AJNR Am J Neuroradiol 2008;29:520-25 CrossRef Medline

28. Hallevi $\mathrm{H}$, Abraham AT, Barreto AD, et al. The spot sign in intracerebral hemorrhage: the importance of looking for contrast extravasation. Cerebrovasc Dis 2010;29:217-20 CrossRef Medline 